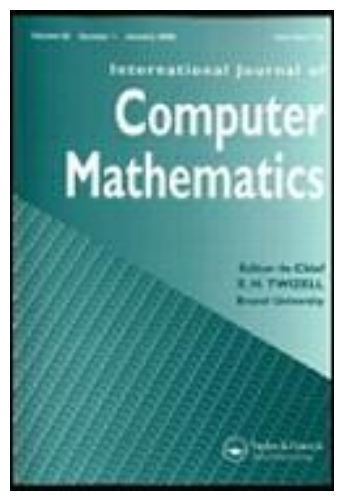

\title{
A virtual pegging approach to the max-min optimization of the bi-criteria knapsack problem
}

\begin{tabular}{|c|c|}
\hline Journal: & International Journal of Computer Mathematics \\
\hline Manuscript ID: & GCOM-2007-0049.R2 \\
\hline Manuscript Type: & Original Article \\
\hline $\begin{array}{r}\text { Date Submitted by the } \\
\text { Author: }\end{array}$ & 12-Aug-2007 \\
\hline Complete List of Authors: & $\begin{array}{l}\text { Taniguchi, Fumiaki; National Defense Academy, Computer Science } \\
\text { Yamada, Takeo; National Defense Academy, Computer Science } \\
\text { Kataoka, Seiji; National Defense Academy, Computer Science }\end{array}$ \\
\hline Keywords: & $\begin{array}{l}\text { Knapsack problem, Bi-objective combinatorial optimization, Pegging } \\
\text { test, } 90 \mathrm{C} 27,65 \mathrm{~K} 05\end{array}$ \\
\hline
\end{tabular}

\section{\scholaroNE" \\ Manuscript Central}




\title{
A virtual pegging approach to the max-min optimization of the bi-criteria knapsack problem
}

\author{
FUMIAKI TANIGUCHI, TAKEO YAMADA* and SEIJI KATAOKA \\ Department of Computer Science, The National Defense Academy, \\ Yokosuka, Kanagawa 239-8686, Japan
}

\begin{abstract}
We are concerned with a variation of the knapsack problem, the bi-objective max-min knapsack problem (BKP), where the values of items differ under two possible scenarios. We give a heuristic algorithm and an exact algorithm to solve this problem. In particular, we introduce a surrogate relaxation to derive upper and lower bounds very quickly, and apply the pegging test to reduce the size of BKP. We also exploit this relaxation to obtain an upper bound in the branch-andbound algorithm to solve the reduced problem. To further reduce the problem size, we propose a 'virtual pegging' algorithm and solve BKP to optimality. As a result, for problems with up to 16000 items we obtain a very accurate approximate solution in less a few seconds. Except for some instances, exact solutions can also be obtained in less than a few minutes on ordinary computers. However, the proposed algorithm is less effective for strongly correlated instances.
\end{abstract}

Keywords: Knapsack problem; Bi-objective combinatorial optimization; Pegging test.

2000 Math Subject Classifications: 90C27; 90C29; 49K35

\footnotetext{
${ }^{*}$ Corresponding author. yamada@nda.ac.jp
} 


\section{Introduction}

Knapsack problem [1, 2] has been studied extensively in operations research and computer science. Although it is an $\mathcal{N} \mathcal{P}$-hard [3] combinatorial optimization problem, it can be solved relatively easily in practice. In this article, we are concerned with a variation of this problem, where the values of items differ under possible two scenarios. By $p_{j}^{k}$ we denote the value of item $j$ under scenario $k(=1,2)$, and $x_{j}$ is the decision variable that takes value 1 if item $j$ is adopted and 0 otherwise for $j=1,2, \ldots, n$. Then,

$$
z^{k}(x):=\sum_{j=1}^{n} p_{j}^{k} x_{j}
$$

is the total value of the solution $x=\left(x_{j}\right)$ under scenario $k$, and thus we have two objective functions to maximize. On the other hand, the weight of item $j$ is assumed to be constant $w_{j}$ through all scenarios, and the knapsack capacity is $c$.

Then, we formulate the bi-objective max-min knapsack problem $[4,5,6]$ as

\section{BKP:}

$$
\begin{array}{ll}
\text { maximize } & \min \left\{\sum_{j=1}^{n} p_{j}^{1} x_{j}, \sum_{j=1}^{n} p_{j}^{2} x_{j}\right\} \\
\text { subject to } & \sum_{j=1}^{n} w_{j} x_{j} \leq c, \\
& x_{j} \in\{0,1\}, \quad j=1,2 \ldots, n .
\end{array}
$$

Without much loss of generality, we assume in the sequel that

$\mathbf{A}_{1}: p_{j}^{k}(j=1,2, \ldots, n ; k=1,2)$ are non-negative integers.

$\mathbf{A}_{2}: w_{j}(j=1,2, \ldots, n)$ and $c$ are positive integers.

$\mathbf{A}_{3}: \sum_{j=1}^{n} w_{j}>c$.

By rewriting BKP as the following equivalent linear integer programming problem, we may solve small instances using free or commercial IP solvers [7].

\section{$\mathrm{BKP}^{\sharp}$ :}

$$
\begin{array}{ll}
\text { maximize } & v \\
\text { subject to } & \sum_{j=1}^{n} p_{j}^{k} x_{j} \geq v, k=1,2,
\end{array}
$$

(3), (4), $v \geq 0$.

Bi-objective knapsack problem has been studied by Eben-Chaime [4] and Zhang et al. [6], who presented parametric or heuristic algorithms to solve the problem. Yu [8], Iida [9] and Kouvelis [5] gave branch-and-bound algorithms for the multi-scenario 
max-min knapsack problem (MKP) respectively, and solved problems with $n \leq 90$ items and 30 scenarios.

In a companion paper [10], we gave reduction and exact algorithms to solve MKP with more than two scenarios. Here, we focus on the case of two scenarios, and present an algorithm that can solve much larger problems than the previous algorithms by employing a virtual pegging approach [11]. First, we introduce in Section 2 the surrogate relaxation to find upper and lower bounds quickly. Then in Section 3, following [10], we introduce a pegging test to reduce the size of the problem, and extend this to the virtual pegging test in Section 4. Through these, the original BKP is reduced (often remarkably) in size, and finally we solve the reduced problem by the 'surrogate relaxation-based' branch-and-bound algorithm of Section 5. Through this approach, we are often able to solve BKPs with up to 16000 items in less than a few minutes. However, in strongly correlated instances, we frequently encounter difficulty in solving small problems with a few hundred items.

\section{Upper and lower bounds}

This section derives an upper bound by applying the surrogate relaxation $[12,8]$ to BKP . At the same time, we obtain an approximate solution, and thus a lower bound to BKP.

\subsection{Surrogate relaxation}

For an arbitrary $\lambda \in[0,1]$ we define the surrogate relaxation of $\mathrm{BKP}$ as follows.

$\operatorname{SBKP}(\lambda)$ :

$$
\begin{array}{ll}
\text { maximize } & \sum_{j=1}^{n} \bar{p}_{j}(\lambda) x_{j} \\
\text { subject to } & \sum_{j=1}^{n} w_{j} x_{j} \leq c, \\
& 0 \leq x_{j} \leq 1, \quad j=1, \ldots, n,
\end{array}
$$

where

$$
\bar{p}_{j}(\lambda):=\lambda p_{j}^{1}+(1-\lambda) p_{j}^{2} .
$$

Here, we note that $x_{j}$ is also relaxed to a continuous variable.

For a fixed $\lambda \in[0,1], \operatorname{SBKP}(\lambda)$ is the continuous knapsack problem whose solution is easily found [2]. Let $\bar{x}(\lambda)=\left(\bar{x}_{j}(\lambda)\right)$ denote an optimal solution to $\operatorname{SBKP}(\lambda)$ with the corresponding optimal value $\bar{z}(\lambda)$, and $z^{\star}$ is the optimal objective value to the original BKP. Then, we have

$$
z^{\star} \leq \bar{z}(\lambda)
$$

i.e., $\bar{z}(\lambda)$ gives an upper bound to BKP.

Analogous to the Lagrangian relaxation $[13,14], \bar{z}(\lambda)$ satisfies the following properties $[\mathbf{1 0}]$. 


\section{Proposition 1}

(i) $\bar{z}(\lambda)$ is a piecewise-linear, convex function of $\lambda$.

(ii) If $\bar{z}(\lambda)$ is differentiable at $\lambda$,

$$
d \bar{z}(\lambda) / d \lambda=z^{1}(\bar{x}(\lambda))-z^{2}(\bar{x}(\lambda)) .
$$

(iii) For $\lambda \in[0,1]$, if $\bar{x}(\lambda)$ is feasible to $\mathrm{BKP}$ and

$$
z^{1}(\bar{x}(\lambda))=z^{2}(\bar{x}(\lambda))
$$

then $\bar{x}(\lambda)$ is an optimal solution to BKP.

\section{$2.2 \quad$ Binary search method}

For an arbitrary $\lambda \in[0,1], \bar{z}(\lambda)$ gives an upper bound to BKP. However, to find an upper bound with $\bar{z}(\lambda)$ as small as possible, we solve the following surrogate dual problem [12]

$$
\begin{array}{ll}
\operatorname{minimize} & \bar{z}(\lambda) \\
\text { subject to } & \lambda \in[0,1] .
\end{array}
$$

Then, taking (11) into account, the following binary search method solves the dual problem.

\section{Algorithm BINARY}

Step 1. Let $\lambda_{L}:=0$ and $\lambda_{R}:=1$.

Step 2. Let $\lambda:=\left(\lambda_{L}+\lambda_{R}\right) / 2$ and solve $\operatorname{SBKP}(\lambda)$ to obtain $\bar{x}(\lambda)$ and $\bar{z}(\lambda)$.

Step 3. If $\lambda_{R}-\lambda_{L}<\epsilon$, or the condition (12) is met, go to Step 5.

Step 4. If $z^{1}(\bar{x}(\lambda))>z^{2}(\bar{x}(\lambda))$ let $\lambda_{R}:=\lambda$, else let $\lambda_{L}:=\lambda$. Go to Step 2.

Step 5. Output $\bar{x}(\lambda)$ and $\bar{z}(\lambda)$, and stop.

Here $\epsilon$ is a sufficiently small 'tolerance limit' of computation, and by $\lambda^{\dagger}$ we denote $\lambda$ upon termination of the above algorithm. Thus, we obtain an optimal upper bound to $\mathrm{BKP}$ as $\bar{z}:=\bar{z}\left(\lambda^{\dagger}\right)$.

\subsection{Lower bounds}

For an arbitrary $\lambda \in[0,1], \bar{x}(\lambda)$ satisfies (3). If this also satisfies the $0-1$ constraint (4), this is feasible to BKP; hence, the corresponding objective value gives a lower bound to the original problem. If, on the other hand, some components of $\bar{x}(\lambda)$ violate (4), we still obtain a feasible solution by replacing all the fractional components with 0 . In BINARY, each time we solve $\operatorname{SBKP}(\lambda)$ we thus get a lower bound, and the largest one found this way gives the best lower bound. This is henceforth denoted as $\underline{z}$. 


\section{Pegging test}

Pegging test $[15,16,17]$ is well known for the ordinary 0-1 knapsack problem. By applying this test, some variables are fixed either at 0 or 1 , and after removing these we obtain a problem of (often significantly) reduced size. In this section, we show that the same pegging test can be applied to BKP by introducing the surrogate relaxation first, as we have shown in [10] for MKP in general.

Assume that we have the optimal surrogate multiplier $\lambda^{\dagger}$, the corresponding upper bound $\bar{z}=\bar{z}\left(\lambda^{\dagger}\right)$ and a lower bound $\underline{z}$ to BKP, and let us consider $\operatorname{SBKP}\left(\lambda^{\dagger}\right)$. In what follows, we write $\bar{p}_{j}:=\bar{p}_{j}\left(\lambda^{\dagger}\right)$ for simplicity. For an arbitrary $u \in\{1,2, \ldots, n\}$, let $\bar{z}_{u, \delta}$ denote the optimal objective value to $\operatorname{SBKP}\left(\lambda^{\dagger}\right)$ with an additional constraint $x_{u}=\delta$, where $\delta$ is either 0 or 1 . Then, if

$$
\bar{z}_{u, 0}<\underline{z}
$$

it is not possible that $x_{u}^{\star}=0$ in any optimal solution $x^{\star}=\left(x_{j}^{\star}\right)$ to BKP, i.e., we necessarily have $x_{u}^{\star}=1$. Similarly, in the case that

$$
\bar{z}_{u, 1}<\underline{z}
$$

$x_{u}^{\star}=0$ must follow.

To determine (13) and (14) quickly, the following shortcut is usually taken. First of all, without loss of generality, we assume the following.

$\mathbf{B}_{1}$ : The items are numbered in the non-increasing order of $\bar{p}_{j} / w_{j}$.

Let $W_{j}$ and $P_{j}$ be, respectively the accumulated weight and profit, i.e.,

$$
W_{j}:=\sum_{i=1}^{j} w_{i}, \quad P_{j}:=\sum_{i=1}^{j} \bar{p}_{i}
$$

where $W_{0}=P_{0}=0$. Then, the broken line connecting $\left\{\left(W_{j}, P_{j}\right) \mid j=0, \cdots, n\right\}$ gives a piecewise-linear, monotonically non-decreasing, concave function [2].

The intersection of this broken line with the vertical line $W=c$ gives an upper bound $\bar{z}$. The item $s$ satisfying $W_{s-1} \leq c<W_{s}$ is said to be the critical item. Here, if for any $u<s$ we set $x_{u}=0$, it is known $[15,16]$ that

$$
\bar{z}_{u, 0} \leq \bar{z}-\theta_{u}
$$

where we define

$$
\theta_{u}:=\bar{p}_{u}-\left(\bar{p}_{s} / w_{s}\right) w_{u}
$$

This is referred to as the threshold for item $u$. Then, we have the following [10]. 
Theorem 1 For any optimal solution $x^{\star}=\left(x_{j}^{\star}\right)$ to BKP, both of the followings hold.

(i) $\bar{z}-\underline{z}<\theta_{j} \Rightarrow x_{j}^{\star}=1$,

(ii) $\bar{z}-\underline{z}<-\theta_{j} \Rightarrow x_{j}^{\star}=0$.

For a pair of upper and lower bounds, by applying this theorem some variables are fixed, and removing these variables we obtain a BKP of (often significantly) reduced size.

\section{Virtual pegging test}

In Theorem 1 we see that the smaller the gap $=\bar{z}-\underline{z}$ between the upper and lower bounds, the more variables are fixed. If the gap is not small enough, the effectiveness of the pegging method is limited, since the size of the problem will not be reduced much in such a case. In the present section, we introduce a virtual pegging test [11], which we originally presented for the precedence constrained knapsack problem, to BKP to cope with this problem.

\subsection{Virtual pegging principle}

In the pegging test based on Theorem 1, the upper and lower bounds necessarily satisfy

$$
\underline{z} \leq z^{\star} \leq \bar{z}
$$

However, we may carry out this test using an arbitrary value $l \leq \bar{z}$ as an 'assumed' lower bound.

Let the set of all the feasible solutions to BKP be $X$, i.e.,

$$
X:=\left\{\left(x_{1}, x_{2}, \ldots, x_{n}\right) \mid \sum_{j=1}^{n} w_{j} x_{j} \leq c, x_{j} \in\{0,1\}, \forall j\right\} .
$$

Then, if we carry out the pegging test (Theorem 1) with $\bar{z}$ and $l$ as upper and lower bounds, some $x_{j}$ 's will be 'fixed' either at 0 or 1 . But this is not necessarily a correct pegging because $l$ is not a guaranteed lower bound to BKP. Let the index sets of variables, which are (temporally) fixed at 0 and 1 by Theorem 1 , be $F_{0}(l)$ and $F_{1}(l)$ respectively. Then, we have the following reduced problem.

$\mathbf{R}(l)$ :

$$
\begin{array}{ll}
\operatorname{maximize} & \min \left\{\sum_{j=1}^{n} p_{j}^{1} x_{j}, \sum_{j=1}^{n} p_{j}^{2} x_{j}\right\} \\
\text { subject to } & x \in X, \\
& x_{j}=0, \forall j \in F_{0}(l), \\
& x_{j}=1, \forall j \in F_{1}(l) .
\end{array}
$$


The optimal objective value to this problem will be denoted as $z_{l}^{\star}$. If $\mathrm{R}(l)$ is infeasible, we define $z_{l}^{\star}:=-\infty$. Then, for the optimal objective value $z^{\star}$ to BKP we have the following [11].

\section{Theorem 2}

(i) $l \leq z^{\star} \Rightarrow z_{l}^{\star}=z^{\star}$.

(ii) $l>z^{\star} \Rightarrow z_{l}^{\star} \leq z^{\star}$.

(iii) $l \leq l^{\prime} \Rightarrow z_{l}^{\star} \geq z_{l^{\prime}}^{\star}$

(iv) $l \leq z_{l}^{\star} \Rightarrow z_{l}^{\star}=z^{\star}$.

As a direct corollary to (iii), if $\mathrm{R}(l)$ is infeasible, then $\mathrm{R}\left(l^{\prime}\right)$ is also infeasible for all $l^{\prime} \geq l$.

\subsection{A virtual pegging algorithm}

For an arbitrary value $l \leq \bar{z}$, by carrying out the virtual pegging test and solving the reduced problem $\mathrm{R}(l)$, we obtain $z_{l}^{\star}$. Then, if (iv) is satisfied in Theorem 2, BKP is solved. In addition, if gap $:=\bar{z}-l$ is small, it is probable that $\mathrm{R}(l)$ is much smaller than the original in size. The reduced $\mathrm{R}(l)$ may be solved by some free or commercial IP solver, but in section 5 we present a branch-and-bound algorithm to solve this problem, and the following algorithm solves BKP completely.

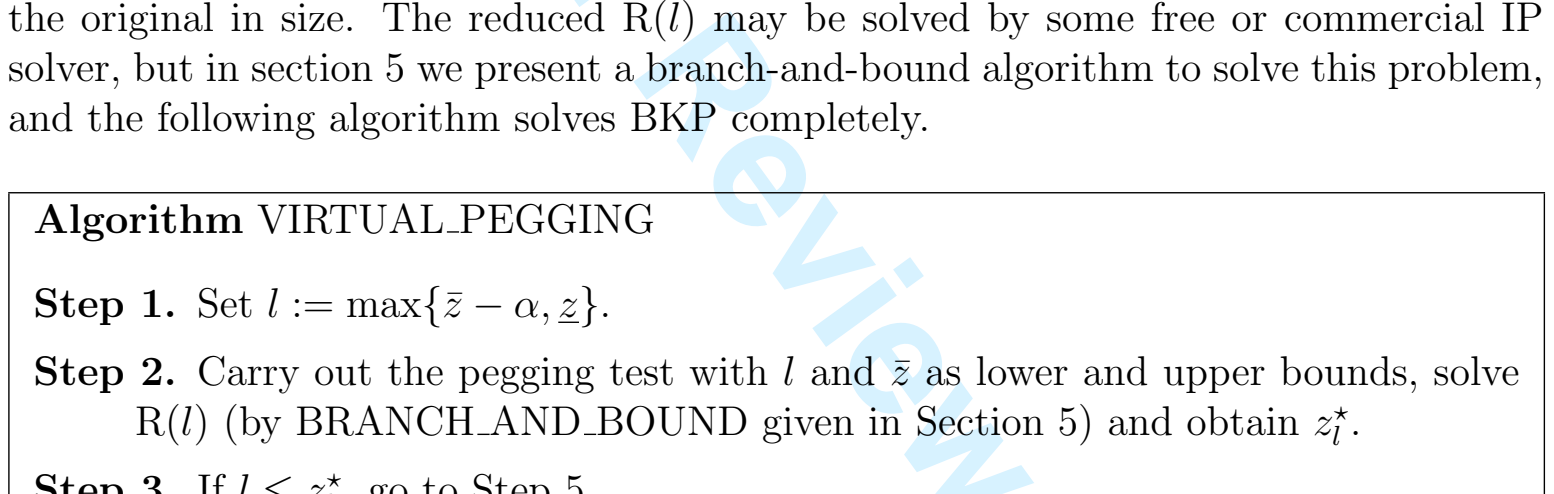

Here, $\alpha$ is an arbitrary small margin between the upper bound and the initially assumed lower bound. We set $l:=\bar{z}-\alpha$ at first if this is not smaller than $\underline{z}$. Then, if the optimal value is not found in Step 3, $l$ is further lowered by $\alpha$, and we repeat Steps $2-4$ all over again until an optimal solution is found.

\section{Surrogate-based branch-and-bound}

For two disjoint subsets $F_{0}$ and $F_{1}$ of $\{1,2, \ldots, n\}$ we consider the subproblem (also referred to as a 'node') of BKP as 
$\mathbf{P}\left(F_{0}, F_{1}\right):$

$$
\begin{array}{ll}
\text { maximize } & \min \left\{\sum_{j=1}^{n} p_{j}^{1} x_{j}, \sum_{j=1}^{n} p_{j}^{2} x_{j}\right\} \\
\text { subject to } & x \in X, \\
& x_{j}=0, \forall j \in F_{0}, \\
& x_{j}=1, \forall j \in F_{1} .
\end{array}
$$

Here $F_{0}\left(F_{1}\right)$ is the set of variables fixed at $0\left(1\right.$, resp.), and by $z^{\star}\left(F_{0}, F_{1}\right)$ we denote the optimal objective value to this problem. Clearly $\mathrm{P}(\emptyset, \emptyset)$ is identical to $\mathrm{BKP}$, and the problem $\mathrm{P}\left(F_{0}(l), F_{1}(l)\right)$ is identical to $\mathrm{R}(l)$.

Next, using $\lambda^{\dagger}$ obtained by BINARY we define its relaxation as

$$
\mathbf{S P}\left(F_{0}, F_{1}\right):
$$

$$
\begin{array}{ll}
\text { maximize } & \sum_{j=1}^{n} \bar{p}_{j} x_{j} \\
\text { subject to } & \sum_{j=1}^{n} w_{j} x_{j} \leq c, \\
& x_{j}=0, \forall j \in F_{0}, \\
& x_{j}=1, \forall j \in F_{1}, \\
& 0 \leq x_{j} \leq 1, \forall j \notin F_{0} \cup F_{1},
\end{array}
$$

and its optimal solution $\bar{x}\left(F_{0}, F_{1}\right)$ with the corresponding objective value $\bar{z}\left(F_{0}, F_{1}\right)$. Note that $\operatorname{SP}\left(F_{0}, F_{1}\right)$ is a continuous knapsack problem which is easily solved. If these are infeasible, we define $z^{\star}\left(F_{0}, F_{1}\right):=-\infty$ and $\bar{z}\left(F_{0}, F_{1}\right):=-\infty$, respectively.

Then, a branch-and-bound algorithm [14] can be constructed as follows. We call BRANCH_AND_BOUND in Step 2 of the VIRTUAL_PEGGING with $F_{0}:=F_{0}(l)$ and $F_{1}:=F_{1}(l)$, and upon termination of this we obtain the optimal objective value as $z_{\mathrm{opt}}^{\star}:=z_{l}^{\star}$. 
Algorithm BRANCH_AND_BOUND $\left(F_{0}, F_{1}\right)$

Step 1. (Initialization) Let the incumbent optimal objective value be $z_{\mathrm{opt}}^{\star}=-\infty$.

Step 2. (Evaluate the current subproblem) Solve $\operatorname{SP}\left(F_{0}, F_{1}\right)$ and obtain $\bar{x}\left(F_{0}, F_{1}\right)$ and $\bar{z}\left(F_{0}, F_{1}\right)$.

Step 3. (Feasible solution) If $\bar{x}\left(F_{0}, F_{1}\right)$ is feasible to $\mathrm{P}\left(F_{0}, F_{1}\right)$, go to Step 6.

Step 4. (Inprospective node) If $\bar{z}\left(F_{0}, F_{1}\right) \leq z_{\text {opt }}^{\star}$, return.

Step 5. (Branch and recursive call) Do the followings.

(i) Find $u:=\min \left\{j \mid j \notin F_{0} \cup F_{1}\right\}$.

(ii) Call BRANCH_AND_BOUND $\left(F_{0} \cup\{u\}, F_{1}\right)$.

(iii) Call BRANCH_AND_BOUND $\left(F_{0}, F_{1} \cup\{u\}\right)$.

(iv) return.

Step 6. (Update incumbent) If $z_{\mathrm{opt}}^{\star}<\bar{z}\left(F_{0}, F_{1}\right)$, update $z_{\mathrm{opt}}^{\star}:=\bar{z}\left(F_{0}, F_{1}\right)$ and return.

By assumption $\mathbf{B}_{1}$ and the definition of $u$ above, the branching is made in the nonincreasing order of $\bar{p}_{j} / w_{j}$ among the unfixed variables. Also, by the recursive structure of the algorithm, subproblems are generated and examined in a depth-first fashion. The characteristic feature of this algorithm is that the upper bound $\bar{z}\left(F_{0}, F_{1}\right)$ can be computed quite rapidly, since $\mathrm{SP}\left(F_{0}, F_{1}\right)$ is a continuous knapsack problem.

\section{A numerical example}

Let us consider BKP with $n=10, c=2500$ and the data of Table 1. Table 2 shows the binary search process, where we obtain $\lambda_{1}^{\dagger}=0.241, \bar{z}=2652.75$ and $\underline{z}=2440$. The gap between the bounds is 212.75 . Table 3 shows the items in the non-increasing order of $\bar{p}_{j} / w_{j}$. Here the critical item is $s=6$, and the thresholds are shown in the row of $\theta_{j}$. The row of $x_{j}^{\star}$ is the result of pegging, where '-' indicates the unfixed variables.

$* * * * * * * * * *$ [[ Insert tables 1-3 about here ]] $* * * * * * * * * *$

Solving this BKP directly by calling BRANCH_AND_BOUND $(\emptyset, \emptyset)$, we obtain the optimal $z^{\star}=2440$ after examining 29 branch-and-bound nodes. If we apply the same method after reducing the problem by the pegging test (Theorem 1) with $\bar{z}-\underline{z}=212.75$, the same solution is obtained after generating 15 subproblems. By the virtual pegging test with $\alpha=100$, we get the solution after examining only 7 subproblems. However, in this case (iv) of Theorem 2 is not satisfied since $l=\bar{z}-\alpha=2552.75>z_{l}^{\star}=2440$, and we need to run VIRTUAL_PEGGING again with $l$ lowered to 2440 to get a guaranteed 
optimal solution. All these solutions coincide with those obtained from NUOPT [18], an MP/IP solver popular in Japan which is considered competitive to such solvers as LINDO, EXPESS-MP, CPLEX, etc. [7].

\section{Numerical experiments}

\subsection{Design of experiments}

For BKP with $n=100-16000$ items, we evaluate the performance of the "surrogate relaxation + (virtual) pegging test + branch-and-bound' approach developed in the previous sections. Weight $w_{j}$ of item $j$ is randomly and unformly distributed over integer interval $[1,1000]$, and the values of items are generated according to

- UNCOR (uncorrelated): $p_{j}^{k}(k=1,2)$ are distributed independently and uniformly over $[1,1000]$,

- WEAK (weakly correlated): $p_{j}^{k}(k=1,2)$ are distributed independently and uniformly over $\left[w_{j}, w_{j}+200\right]$,

- STRONG (strongly correlated): $p_{j}^{1}:=w_{j}+100$, and $p_{j}^{2}$ is distributed uniformly over $\left[w_{j}, w_{j}+200\right]$.

Knapsack capacity is set to

$$
c:=500 n \cdot \rho
$$

where $\rho$ is either $0.25,0.50$ or 0.75 . Since the average weight of an item is $500.5, \rho=0.50$ means that approximately a half of all items can be accommodated in the knapsack.

To compute surrogate relaxation, pegging test and BRANCH_AND_BOUND, we have implemented the algorithm in ANSI C language on an IBM RS/6000 SP 44 Model 270 workstation (CPU: POWER 3-II, 375Mhz). We also solved small instances using NUOPT Ver. 3.3.0 on the same machine.

\subsection{Bounds and reduction}

Tables 4 and 5 give the results of computation of the upper and lower bounds, as well as of the pegging test. Here, in addition to the bounds $\bar{z}$ and $\underline{z}$, we show the gap $(\bar{z}-\underline{z})$, the relative error defined by

$$
\operatorname{rerror}(\%)=100 \cdot(\bar{z}-\underline{z}) / \underline{z},
$$

the number of unfixed variables $\left(n^{\prime}\right)$, and the $\mathrm{CPU}$ time in seconds $\left(\mathrm{CPU}_{0}\right)$ to evaluate the bounds and the effect of the pegging test. Each row is the average over 10 randomly generated instances.

From these tables, we observe the following.

1. By the surrogate relaxation we obtain an upper bound and a heuristic solution of very high precision in a few CPU seconds. 
2. Gaps are smaller in WEAK case, but the reduced problems are of almost comparable size. In both of UNCOR and WEAK cases, problems are reduced considerably in size.

3. $\mathrm{CPU}_{0}$ increases with $n$, but this is rather insensitive to the correlation type of instances.

4. The objective value ( $\bar{z}$ and $\underline{z}$ ) increases with $\rho$, but no significant influence of $\rho$ is seen on the accuracy of heuristic solutions or CPU time.

*********** [[ Insert tables 4-5 about here ]] $* * * * * * * * * *$

\subsection{Exact solution}

To solve BKP exactly, we compare the following three methods.

- PEG-NUOPT: Apply the pegging test (Theorem 1) to BKP, and solve the reduced problem using NUOPT.

- PEG-BAB: Apply the same pegging test, and then solve the reduced problem by calling BRANCH_AND_BOUND.

- VPEG-BAB: Solve BKP by calling VIRTUAL_PEGGING with $l:=\bar{z}-\alpha$.

Tables 6 and 7 compare PEG-NUOPT against PEG-BAB. For each value of $\rho$ and $n$, we computed the randomly generated 10 instances, with the computation truncated at the time limit of TL=1800 seconds. The column of '\#sol' show the number of runs completed within TL, and all the rows are the average over the finished instances. In all solved cases, we obtained the identical objective values. In these tables, ' $\mathrm{BBN}$ ' is the number (in millions) of the branch-and-bound nodes generated, and 'CPU' is the time in seconds to solve the problem completely by respective methods.

*********** [[ Insert tables 6-7 about here ]] **********

The observation from these tables are:

1. PEG-NUOPT was able to solve problems of $n=2000$ or smaller, but for larger $n$ it often fails; especially for $n \geq 10000$ this approach is almost hopeless irrespective to $\rho$ and correlation type of instances.

2. PEG-BAB solved all problems with $n \leq 6000$, and frequently solved larger problems. No clear difference was seen in the difficulty of solving UNCOR and WEAK instances. 


\subsection{Virtual pegging result}

VPEG-BAB was run with the initial margin

$$
\alpha:=1000 \cdot \log ^{2} n / n
$$

This follows Lueker [19], who proved for the 0-1 knapsack problem (KP)

$$
\bar{z}_{\mathrm{KP}}-z_{\mathrm{KP}}^{\star}=O\left(\log ^{2} n / n\right)
$$

where $\bar{z}_{\mathrm{KP}}$ and $z_{\mathrm{KP}}^{\star}$ are, respectively, the continuously relaxed upper bound and the optimal objective value. After some preliminary experiments, we set the constant in (20) as 1000. In all our computation with (20),

$$
\bar{z}-\alpha<z^{\star}
$$

was confirmed a posteriori, and thus $\bar{z}-\alpha$ was indeed a correct lower bound in all instances tested. Therefore, in VIRTUAL_PEGGING Steps 2 through 4 were actually repeated only once. The results are shown in Tables 8 and 9 , where in addition to $\mathrm{BBN}, \mathrm{CPU}$ and \#sol, the number of unfixed variables by virtual pegging $\left(n^{\prime \prime}\right)$, and the optimal objective value $\left(z^{\star}\right)$ are shown.

************ [[ Insert table 8-9 about here ]] **********

Except for a few correlated instances, almost all problems were solved within a few minutes. Comparing Tables 6 and 8 (also 7 and 9), the effectiveness of the virtual pegging approach is evident.

\subsection{Comparison against other commercial solvers}

We further evaluate PEG-BAB against two leading IP solvers CPLEX 10.1 [20] and XPRESS-MP release 2005B [21] on an even faster computer DELL Dimension 8400 (Pentium(R) $4 \mathrm{CPU}, 3.40 \mathrm{GHz}$ ). Table 10 gives the summary of CPU times in solving UNCOR and WEAK instances with $\rho=0.25$ and $n=2000,4000,8000$. Ten instances solved in each row are identical to those in the corresponding rows of Tables 6 and 7 . Here the columns show the CPU time in seconds of the following solution methods:

- CPLEX: Solve BKP $\sharp$ directly by using CPLEX.

- PEG-CPLEX: Apply the pegging test, and then solve the reduced problem by CPLEX.

- PEG-XPRESS: Also, solve the reduced problem by XPRESS-MP. 
************ [[ Insert table 10 about here ]] $* * * * * * * * * *$

We observe the pegging test effective as a pre-processing in solving the problem, and PEG-BAB is substantially advantageous to these commercial solvers.

\subsection{Small and strongly correlated instances}

In standard 0-1 knapsack problems, it is known that small instances are often harder to solve to optimality than the larger ones [22]. Table 11 summerizes the result of experiments for BKP with $n$ between 100-500, where we compare PEG-CPLEX and VPEG-BAB. As in previous tables, each row is the average over the solved cases out of 10 randomly generated instances, and $n^{\prime \prime}$ is the number of variables in the reduced problem.

From this, we observe that UNCOR and WEAK instances of this size are easily solved by both CPLEX and VPEG-BAB. On the other hand, in STRONG we often encounter instances that can not be solved within the fixed time limit of 1800 seconds, while some instances are solved within a few seconds. Especially, in STRONG case the virtual pegging method becomes less effective as $n$ increases, and this makes VPEG-BAB unsatisfactory in such a case.

$* * * * * * * * * * *[[$ Insert table 11 about here $]]$ **********

\section{Conclusion}

We gave heuristic and exact algorithms to solve BKP. In addition to the surrogate relaxation, the pegging test and the surrogate-based branch-and-bound method, we presented a virtual pegging algorithm. For problems with $n \leq 16000$ we were able to obtain approximate solutions of quite high accuracy in less than a few seconds, and except for some instances, exact solutions were obtained by VPEG-BAB in less than a few minutes on an ordinary computer. However, strongly correlated instances were difficult to solve by this method, unless the problem size was very small.

Acknowledgements The authors are greatful to anonymous referees for their careful reading of the manuscript and helpful comments. 


\section{References}

[1] Kellerer, H., Pferschy, U. and Pisinger, D., 2004, Knapsack Problems(Berlin: Springer).

[2] Martello, S. and Toth, P., 1990, Knapsack Problems: Algorithms and Computer Implementations, (New York: Wiley).

[3] Garey, M.R. and Johnson, D.S., 1979, Computers and Intractability: A Guide to the Theory of NP-Completeness(New York: Freeman and Company).

[4] Eben-Chaime, M., 1996, Parametric solution for linear bicriteria knapsack models. Management Science, 42, 1565-1575.

[5] Kouvelis, P. and Yu, G., 1997, Robust Discrete Optimization and Its Applications(Dordrecht: Kluwer).

[6] Zhang, C.-N. and Ong, H.-L., 2004, Solving the bicriteria zero-one knapsack problem by an efficient LP-based heuristic. European Journal of Operational Research, 159, 545-557.

[7] Fourer, R., 1999, Software survey: linear programming. OR/MS Today, 26, 64-71.

[8] Yu, G., 1996, On the max-min 0-1 knapsack problem with robust optimization applications. Operations Research, 44, 407-415.

[9] Iida, H., 1999, A note on the max-min 0-1 knapsack problem. Journal of Combinatorial Optimization, 3, 89-94.

[10] Taniguchi, F., Yamada, T. and Kataoka, S., 2007, Heuristic and exact algorithms for the max-min optimization of the multi-criteria knapsack problem. to appear in Computers $\&$ Operations Research.

[11] You, B.-J. and Yamada, T., 2007, A virtual pegging approach to the precedence constrained knapsack problem. European J. Operational Research, 183, 618-632.

[12] Glover, F., 1975, Surrogate constraint duality in mathematical programming. Operations Research, 23, 434-451.

[13] Fisher, M., 2004, The Lagrangian relaxation method for solving integer programming problems. Management Science, 50, 1861-1871.

[14] Wolsey, L.A., 1998, Integer Programming(New York: Wiley).

[15] Dembo, R.S. and Hammer, P.L., 1980, A reduction algorithm for knapsack problems. Methods of Operations Research, 36, 49-60. 
[16] Fayard, D. and Plateau, G., 1975, Resolution of the 0-1 knapsack problem: comparison of methods. Mathematical Programming, 8, 272-307.

[17] Ingargiola, G.P. and Korsh, F.F., 1973, Reduction algorithms for zero-one single knapsack problems. Management Science, 20, 460-463.

[18] NUOPT Ver. 3.3.0, 2002, http://www.msi.co.jp/nuopt.

[19] Lueker, G., 1982, On the average difference between the solutions to linear and integer knapsack problems. Applied Probability-Computer Science, The Interface I, 489-504.

[20] CPLEX 10.0, ILOG, 2007, http://www.ilog.com/products/cplex/news/whatsnew. cfm.

[21] XPRESS-MP release 2005B, Dash Optimization, 2005, http://www.dashoptimization.com.

[22] Balas, E. and Zemel, E., 1980, An algorithm for large zero-one knapsack problems. Operations Research, 28, 1130-1154. 
Table 1: Data for the example of Section 6.

\begin{tabular}{rrrrrrrrrrr}
\hline$j$ & 1 & 2 & 3 & 4 & 5 & 6 & 7 & 8 & 9 & 10 \\
\hline$w_{j}$ & 618 & 595 & 586 & 427 & 695 & 816 & 833 & 624 & 353 & 611 \\
$p_{j}^{1}$ & 298 & 97 & 24 & 866 & 936 & 252 & 135 & 535 & 386 & 399 \\
$p_{j}^{2}$ & 274 & 102 & 129 & 433 & 859 & 674 & 114 & 407 & 561 & 212 \\
\hline
\end{tabular}


Table 2: Binary search process to find $\lambda^{\dagger}$.

\begin{tabular}{rcccccc}
\hline cycle & $\lambda_{\mathrm{L}}$ & $\lambda_{\mathrm{R}}$ & $\lambda_{1}$ & $\bar{z}(\lambda)$ & $\underline{z}(\lambda)$ & $z^{1}-z^{2}$ \\
\hline 0 & 0.000 & 1.000 & 0.500 & 2719.03 & 2260 & + \\
1 & 0.000 & 0.500 & 0.250 & 2655.12 & 2260 & + \\
2 & 0.000 & 0.250 & 0.125 & 2657.80 & 2440 & - \\
3 & 0.125 & 0.250 & 0.188 & 2655.04 & 2440 & - \\
4 & 0.188 & 0.250 & 0.219 & 2653.67 & 2440 & - \\
5 & 0.219 & 0.250 & 0.234 & 2652.98 & 2440 & - \\
6 & 0.234 & 0.250 & 0.242 & 2653.12 & 2260 & + \\
7 & 0.234 & 0.242 & 0.238 & 2652.80 & 2440 & - \\
8 & 0.238 & 0.242 & 0.240 & 2652.72 & 2440 & - \\
9 & 0.240 & 0.242 & 0.241 & 2652.88 & 2260 & + \\
10 & 0.240 & 0.241 & 0.241 & 2652.75 & 2260 & + \\
\hline
\end{tabular}


Table 3: Threshold $\theta_{j}$ and pegging result.

\begin{tabular}{crrrrrrrrrr}
\hline$j$ & 9 & 5 & 4 & 8 & 6 & 1 & 10 & 3 & 2 & 7 \\
\hline$w_{j}$ & 353 & 695 & 427 & 624 & 816 & 618 & 611 & 586 & 595 & 833 \\
$\bar{p}_{j}\left(\lambda^{\dagger}\right)$ & 518.9 & 877.5 & 537.2 & 437.8 & 572.4 & 279.8 & 257.0 & 103.7 & 100.8 & 119.8 \\
$\theta_{j}$ & 271.2 & 390.0 & 237.7 & 0.1 & 0.0 & -153.7 & -171.6 & -307.3 & -316.6 & -465.3 \\
$x_{j}^{\star}$ & 1 & 1 & 1 & - & - & - & - & 0 & 0 & 0 \\
\hline
\end{tabular}


Table 4: Upper and lower bounds and pegging result (UNCOR case).

\begin{tabular}{rrrrrrrr}
\hline \multicolumn{1}{c}{$n$} & \multicolumn{1}{c}{$\bar{z}$} & \multicolumn{1}{c}{$\underline{z}$} & \multicolumn{1}{c}{ gap } & rerror (\%) & \multicolumn{1}{c}{$n^{\prime}$} & CPU $_{0}$ \\
\hline 0.25 & 2000 & 539586.4 & 539419.6 & 166.8 & 0.031 & 609.1 & 0.06 \\
& 4000 & 1079071.4 & 1078929.9 & 141.5 & 0.013 & 1043.2 & 0.16 \\
& 6000 & 1624944.1 & 1624749.8 & 194.3 & 0.012 & 2090.5 & 0.42 \\
& 8000 & 2154276.5 & 2154148.5 & 128.0 & 0.006 & 1848.7 & 0.52 \\
& 10000 & 2690716.2 & 2690584.6 & 131.6 & 0.005 & 2414.9 & 0.64 \\
& 12000 & 3230946.9 & 3230737.7 & 209.2 & 0.006 & 4397.7 & 1.53 \\
& 14000 & 3771746.4 & 3771598.3 & 148.1 & 0.004 & 3714.2 & 1.40 \\
& 16000 & 4312959.8 & 4312774.5 & 185.3 & 0.004 & 5194.8 & 2.39 \\
\hline 0.50 & 2000 & 763914.3 & 763752.9 & 161.4 & 0.021 & 690.5 & 0.07 \\
& 4000 & 1525945.2 & 1525821.5 & 123.7 & 0.008 & 1101.2 & 0.26 \\
& 6000 & 2290905.1 & 2290762.2 & 142.9 & 0.006 & 1721.7 & 0.41 \\
& 8000 & 3043770.1 & 3043450.6 & 319.5 & 0.010 & 4697.2 & 1.47 \\
& 10000 & 3803532.2 & 3803369.6 & 162.6 & 0.004 & 3591.2 & 1.32 \\
& 12000 & 4561522.5 & 4561323.5 & 199.0 & 0.004 & 4671.0 & 2.29 \\
& 14000 & 5328017.5 & 5327728.5 & 289.0 & 0.005 & 7073.0 & 4.26 \\
& 16000 & 6089334.2 & 6089105.5 & 228.7 & 0.004 & 7195.4 & 4.00 \\
\hline 0.75 & 2000 & 918868.2 & 918757.5 & 110.7 & 0.012 & 435.6 & 0.05 \\
& 4000 & 1838039.1 & 1837938.1 & 101.0 & 0.005 & 798.4 & 0.23 \\
& 6000 & 2754998.5 & 2754871.3 & 127.2 & 0.005 & 1412.7 & 0.50 \\
& 8000 & 3665178.5 & 3665075.7 & 102.8 & 0.003 & 1657.0 & 0.38 \\
& 10000 & 4579119.1 & 4579022.7 & 96.4 & 0.002 & 1913.0 & 1.02 \\
& 12000 & 5490787.8 & 5490581.2 & 206.6 & 0.004 & 4365.2 & 2.23 \\
& 14000 & 6413209.0 & 6413037.1 & 171.9 & 0.003 & 3938.6 & 3.02 \\
& 16000 & 7326788.9 & 7326515.2 & 273.7 & 0.004 & 6804.6 & 5.32 \\
\hline
\end{tabular}


Table 5: Upper and lower bounds and pegging result (WEAK case)

\begin{tabular}{rrrrrcrc}
\hline$\rho$ & \multicolumn{1}{c}{$n$} & \multicolumn{1}{c}{$\bar{z}$} & \multicolumn{1}{c}{ gap } & rerror $(\%)$ & $n^{\prime}$ & $\mathrm{CPU}_{0}$ \\
\hline 0.25 & 2000 & 357159.9 & 357120.3 & 39.6 & 0.001 & 720.7 & 0.08 \\
& 4000 & 714453.0 & 714417.4 & 35.6 & 0.005 & 1204.7 & 0.21 \\
& 6000 & 1072339.1 & 1072307.9 & 31.2 & 0.003 & 1724.4 & 0.51 \\
& 8000 & 1429168.4 & 1429140.6 & 27.8 & 0.002 & 2089.0 & 0.57 \\
& 10000 & 1786782.7 & 1786748.8 & 33.9 & 0.002 & 3078.3 & 0.90 \\
& 12000 & 2143235.6 & 2143196.7 & 38.9 & 0.002 & 4288.6 & 1.71 \\
& 14000 & 2501692.2 & 2501650.9 & 41.3 & 0.002 & 5220.0 & 2.42 \\
& 16000 & 2859882.9 & 2859830.3 & 52.6 & 0.002 & 7239.7 & 3.82 \\
\hline 0.50 & 2000 & 651452.4 & 651415.3 & 37.1 & 0.006 & 805.4 & 0.10 \\
& 4000 & 1302535.2 & 1302488.6 & 46.6 & 0.004 & 1918.8 & 0.39 \\
& 6000 & 1954819.6 & 1954775.3 & 44.3 & 0.002 & 2667.3 & 0.58 \\
& 8000 & 2606223.9 & 2606167.5 & 56.4 & 0.002 & 4530.3 & 1.44 \\
& 10000 & 3257987.2 & 3257950.2 & 37.0 & 0.001 & 4064.5 & 1.84 \\
& 12000 & 3908854.0 & 3908793.7 & 60.3 & 0.002 & 7088.5 & 3.34 \\
& 14000 & 4561511.9 & 4561454.2 & 57.7 & 0.001 & 7603.3 & 4.82 \\
& 16000 & 5213789.2 & 5213744.0 & 45.2 & 0.001 & 7604.7 & 4.99 \\
\hline 0.75 & 2000 & 932043.5 & 931997.9 & 45.6 & 0.005 & 849.1 & 0.14 \\
& 4000 & 1863882.4 & 1863840.3 & 42.1 & 0.002 & 1648.4 & 0.44 \\
& 6000 & 2796998.2 & 2796951.7 & 46.5 & 0.002 & 2555.2 & 0.73 \\
& 8000 & 3729220.2 & 3729178.1 & 42.1 & 0.001 & 3199.7 & 1.07 \\
& 10000 & 4662129.1 & 4662087.8 & 41.3 & 0.001 & 3790.4 & 2.34 \\
& 12000 & 5593840.7 & 5593811.9 & 28.8 & 0.001 & 3472.7 & 3.00 \\
14000 & 6527118.2 & 6527070.5 & 47.7 & 0.001 & 6112.9 & 3.91 \\
& 16000 & 7460437.6 & 7460387.5 & 50.1 & 0.001 & 7433.5 & 5.87 \\
\hline
\end{tabular}


Table 6: Exact solution (UNCOR case).

\begin{tabular}{|c|c|c|c|c|c|c|}
\hline \multirow[b]{2}{*}{$\rho$} & \multirow[b]{2}{*}{$n$} & \multicolumn{2}{|c|}{ PEG-NUOPT } & \multicolumn{3}{|c|}{ PEG-BAB } \\
\hline & & $\mathrm{CPU}$ & \#sol & $\mathrm{BBN}\left(\times 10^{6}\right)$ & $\mathrm{CPU}$ & \#sol \\
\hline \multirow[t]{8}{*}{0.25} & 2000 & 90.1 & 10 & 3.02 & 1.31 & 10 \\
\hline & 4000 & 383.5 & 10 & 11.98 & 5.03 & 10 \\
\hline & 6000 & 478.6 & 4 & 68.69 & 28.33 & 10 \\
\hline & 8000 & 608.2 & 3 & 22.48 & 9.72 & 10 \\
\hline & 10000 & - & 0 & 318.88 & 129.68 & 9 \\
\hline & 12000 & - & 0 & 60.19 & 25.49 & 6 \\
\hline & 14000 & 1129.2 & 1 & 142.23 & 59.02 & 9 \\
\hline & 16000 & 1100.5 & 2 & 618.84 & 254.49 & 9 \\
\hline \multirow[t]{8}{*}{0.50} & 2000 & 148.1 & 10 & 4.01 & 1.73 & 10 \\
\hline & 4000 & 630.4 & 5 & 19.47 & 8.21 & 10 \\
\hline & 6000 & 752.7 & 4 & 200.81 & 81.57 & 10 \\
\hline & 8000 & 828.1 & 1 & 105.55 & 45.00 & 8 \\
\hline & 10000 & 488.3 & 1 & 165.03 & 68.55 & 10 \\
\hline & 12000 & 871.9 & 1 & 529.60 & 218.42 & 8 \\
\hline & 14000 & & 0 & 38.48 & 16.18 & 3 \\
\hline & 16000 & - & 0 & 101.27 & 229.45 & 8 \\
\hline \multirow[t]{8}{*}{0.75} & 2000 & 93.3 & 10 & 2.79 & 1.22 & 10 \\
\hline & 4000 & 418.8 & 8 & 6.79 & 3.01 & 10 \\
\hline & 6000 & 225.8 & 4 & 13.69 & 6.21 & 10 \\
\hline & 8000 & 705.1 & 6 & 64.44 & 26.50 & 8 \\
\hline & 10000 & 535.8 & 2 & 32.57 & 14.18 & 9 \\
\hline & 12000 & 1119.1 & 1 & 65.21 & 29.17 & 7 \\
\hline & 14000 & - & 0 & 29.55 & 13.60 & 8 \\
\hline & 16000 & 1078.4 & 1 & 61.65 & 28.15 & 5 \\
\hline
\end{tabular}


Table 7: Exact solution (WEAK case).

\begin{tabular}{|c|c|c|c|c|c|c|}
\hline \multirow[b]{2}{*}{$\rho$} & \multirow[b]{2}{*}{$n$} & \multicolumn{2}{|c|}{ PEG-NUOPT } & \multicolumn{3}{|c|}{ PEG-BAB } \\
\hline & & $\mathrm{CPU}$ & $\#$ sol & $\mathrm{BBN}\left(\times 10^{6}\right)$ & $\mathrm{CPU}$ & \#sol \\
\hline \multirow[t]{8}{*}{0.25} & 2000 & 222.3 & 10 & 5.48 & 2.31 & 10 \\
\hline & 4000 & 616.5 & 6 & 7.84 & 3.43 & 10 \\
\hline & 6000 & 725.5 & 2 & 61.53 & 25.50 & 10 \\
\hline & 8000 & 1537.9 & 2 & 18.49 & 8.13 & 10 \\
\hline & 10000 & - & 0 & 19.73 & 8.83 & 8 \\
\hline & 12000 & - & 0 & 201.81 & 84.09 & 10 \\
\hline & 14000 & 934.0 & 1 & 23.67 & 12.53 & 10 \\
\hline & 16000 & - & 0 & 127.45 & 309.85 & 7 \\
\hline \multirow[t]{8}{*}{0.50} & 2000 & 545.9 & 10 & 5.87 & 2.51 & 10 \\
\hline & 4000 & 693.5 & 3 & 24.90 & 10.60 & 10 \\
\hline & 6000 & 761.4 & 3 & 108.03 & 44.83 & 10 \\
\hline & 8000 & 694.9 & 1 & 17.81 & 9.46 & 7 \\
\hline & 10000 & - & 0 & 36.42 & 17.06 & 9 \\
\hline & 12000 & - & 0 & 591.22 & 244.76 & 8 \\
\hline & 14000 & - & 0 & 727.68 & 303.20 & 9 \\
\hline & 16000 & - & 0 & 110.93 & 51.25 & 8 \\
\hline \multirow[t]{8}{*}{0.75} & 2000 & 399.7 & 10 & 7.07 & 3.05 & 10 \\
\hline & 4000 & 978.8 & 4 & 13.89 & 6.15 & 10 \\
\hline & 6000 & 218.4 & 1 & 226.01 & 92.21 & 10 \\
\hline & 8000 & 1574.7 & 1 & 24.62 & 11.13 & 9 \\
\hline & 10000 & 998.6 & 3 & 25.32 & 13.17 & 8 \\
\hline & 12000 & - & 0 & 23.77 & 12.85 & 8 \\
\hline & 14000 & 1208.0 & 2 & 171.23 & 74.40 & 9 \\
\hline & 16000 & - & 0 & 154.53 & 70.37 & 8 \\
\hline
\end{tabular}


Table 8: Exact solution by VPEG-BAB (UNCOR case).

\begin{tabular}{crrrrrrr}
\hline$\rho$ & \multicolumn{1}{c}{$n$} & \multicolumn{1}{c}{$\alpha$} & \multicolumn{1}{c}{$n^{\prime \prime}$} & \multicolumn{1}{c}{$z^{\star}$} & $\mathrm{BBN}\left(\times 10^{6}\right)$ & $\mathrm{CPU}$ & \#sol \\
\hline 0.25 & 2000 & 28.89 & 115.7 & 539572.2 & 1.24 & 0.55 & 10 \\
& 4000 & 17.20 & 131.8 & 1079062.3 & 2.89 & 1.27 & 10 \\
& 6000 & 12.61 & 137.5 & 1624937.1 & 10.64 & 4.48 & 10 \\
& 8000 & 10.10 & 148.9 & 2154272.2 & 5.04 & 2.22 & 10 \\
& 10000 & 8.48 & 158.3 & 2690712.3 & 40.07 & 16.45 & 10 \\
& 12000 & 7.35 & 167.1 & 3230943.6 & 38.94 & 16.03 & 10 \\
& 14000 & 6.51 & 173.7 & 3771743.7 & 17.11 & 7.26 & 10 \\
& 16000 & 5.86 & 174.0 & 4312957.7 & 17.58 & 7.49 & 10 \\
\hline 0.50 & 2000 & 28.89 & 132.7 & 763902.3 & 1.72 & 0.75 & 10 \\
& 4000 & 17.20 & 167.1 & 1525938.1 & 7.52 & 3.13 & 10 \\
& 6000 & 12.61 & 183.6 & 2290900.8 & 12.04 & 5.01 & 10 \\
& 8000 & 10.10 & 192.6 & 3043766.6 & 17.92 & 7.43 & 10 \\
& 10000 & 8.48 & 207.2 & 3803529.5 & 14.19 & 5.94 & 10 \\
& 12000 & 7.35 & 211.0 & 4561519.8 & 25.63 & 10.62 & 10 \\
& 14000 & 6.51 & 218.4 & 5328015.1 & 391.06 & 160.32 & 10 \\
& 16000 & 5.86 & 229.6 & 6089332.0 & 44.27 & 18.24 & 10 \\
\hline 0.75 & 2000 & 28.89 & 118.7 & 918854.7 & 1.64 & 0.72 & 10 \\
& 4000 & 17.20 & 140.9 & 1838031.7 & 2.49 & 1.09 & 10 \\
& 6000 & 12.61 & 159.4 & 2754994.0 & 5.72 & 2.46 & 10 \\
& 8000 & 10.10 & 170.1 & 3665174.7 & 35.04 & 14.46 & 10 \\
& 10000 & 8.48 & 170.1 & 4579116.2 & 19.10 & 7.94 & 10 \\
& 12000 & 7.35 & 177.2 & 5490784.4 & 65.82 & 27.89 & 10 \\
14000 & 6.51 & 185.5 & 6413206.3 & 48.47 & 19.99 & 10 \\
16000 & 5.86 & 190.8 & 7326786.7 & 189.54 & 81.75 & 10 \\
\hline & & & & & & &
\end{tabular}


Table 9: Exact solution by VPEG-BAB (WEAK case).

\begin{tabular}{rrrrrrrr}
\hline \multicolumn{1}{c}{$n$} & \multicolumn{1}{c}{$\alpha$} & \multicolumn{1}{c}{$n^{\prime \prime}$} & \multicolumn{1}{c}{$z^{\star}$} & $\mathrm{BBN}\left(\times 10^{6}\right)$ & $\mathrm{CPU}$ & \#sol \\
\hline 0.25 & 2000 & 28.89 & 500.0 & 357157.3 & 4.55 & 1.85 & 10 \\
& 4000 & 17.20 & 602.4 & 714451.8 & 5.15 & 2.13 & 10 \\
& 6000 & 12.61 & 694.0 & 1072338.4 & 14.40 & 5.86 & 10 \\
& 8000 & 10.10 & 755.7 & 1429167.8 & 7.28 & 3.07 & 10 \\
& 10000 & 8.48 & 788.1 & 1786782.0 & 91.46 & 37.46 & 10 \\
& 12000 & 7.35 & 821.2 & 2143235.3 & 10.64 & 4.51 & 10 \\
& 14000 & 6.51 & 836.3 & 2501691.6 & 6.29 & 2.83 & 10 \\
& 16000 & 5.86 & 879.8 & 2859882.3 & 160.19 & 238.81 & 10 \\
\hline 0.50 & 2000 & 28.89 & 629.0 & 651449.6 & 5.43 & 2.21 & 10 \\
& 4000 & 17.20 & 811.8 & 1302534.1 & 8.81 & 3.58 & 10 \\
& 6000 & 12.61 & 890.8 & 1954818.7 & 12.01 & 4.91 & 10 \\
& 8000 & 10.10 & 940.7 & 2606223.1 & 588.09 & 377.09 & 10 \\
& 10000 & 8.48 & 991.2 & 3257986.8 & 10.20 & 4.27 & 10 \\
& 12000 & 7.35 & 1020.6 & 3908853.7 & 160.21 & 64.09 & 10 \\
& 14000 & 6.51 & 1058.3 & 4561511.8 & 157.68 & 62.61 & 10 \\
& 16000 & 5.86 & 1110.8 & 5214818.3 & 41.33 & 16.70 & 8 \\
\hline 0.75 & 2000 & 28.89 & 564.1 & 932040.4 & 6.95 & 2.82 & 10 \\
& 4000 & 17.20 & 699.5 & 1863881.2 & 9.39 & 3.82 & 10 \\
& 6000 & 12.61 & 738.7 & 2796997.4 & 140.28 & 56.95 & 10 \\
& 8000 & 10.10 & 810.2 & 3729219.5 & 29.56 & 11.99 & 10 \\
10000 & 8.48 & 843.6 & 4662087.1 & 161.29 & 66.85 & 9 \\
12000 & 7.35 & 891.3 & 5594031.2 & 376.50 & 149.46 & 9 \\
14000 & 6.51 & 918.3 & 6527118.0 & 72.05 & 28.84 & 10 \\
16000 & 5.86 & 930.2 & 7460150.6 & 30.00 & 12.29 & 9 \\
\hline & & & & & & &
\end{tabular}


Table 10: CPU seconds of IP solvers vs. PEG-BAB for instances with $\rho=0.25$.

\begin{tabular}{rcrrrr}
\hline Problem & $n$ & CPLEX & PEG-CPLEX & PEG-XPRESS & PEG-BAB \\
\hline UNCOR & 2000 & 15.46 & 6.01 & 9.64 & 0.41 \\
& 4000 & 55.33 & 16.29 & 21.61 & 1.57 \\
& 8000 & 266.31 & 81.59 & 87.33 & 3.11 \\
\hline \multirow{2}{*}{ WEAK } & 2000 & 49.11 & 21.21 & 21.13 & 0.75 \\
& 4000 & 66.83 & 22.55 & 31.86 & 1.19 \\
& 8000 & 147.53 & 62.32 & 167.18 & 10.80 \\
\hline
\end{tabular}


Table 11: Small size instances.

\begin{tabular}{|c|c|c|c|c|c|c|}
\hline \multirow[b]{2}{*}{ Problem } & \multirow[b]{2}{*}{$n$} & \multicolumn{2}{|c|}{ PEG-CPLEX } & \multicolumn{3}{|c|}{ VPEG-BAB } \\
\hline & & $\mathrm{CPU}$ & $\#$ sol & $n^{\prime \prime}$ & $\overline{\mathrm{CPU}}$ & $\#$ sol \\
\hline \multirow[t]{5}{*}{ UNCOR } & 100 & 0.06 & 10 & 31.8 & 0.00 & 10 \\
\hline & 200 & 0.12 & 10 & 43.9 & 0.00 & 10 \\
\hline & 300 & 0.40 & 10 & 58.7 & 0.01 & 10 \\
\hline & 400 & 0.81 & 10 & 65.3 & 0.01 & 10 \\
\hline & 500 & 0.56 & 10 & 71.0 & 0.01 & 10 \\
\hline \multirow[t]{5}{*}{ WEAK } & 100 & 0.16 & 10 & 81.9 & 0.00 & 10 \\
\hline & 200 & 0.57 & 10 & 149.8 & 0.01 & 10 \\
\hline & 300 & 1.60 & 10 & 191.6 & 0.05 & 10 \\
\hline & 400 & 1.23 & 10 & 171.3 & 0.06 & 10 \\
\hline & 500 & 2.17 & 10 & 317.0 & 0.06 & 10 \\
\hline \multirow[t]{5}{*}{ STRONG } & 100 & 0.22 & 10 & 64.0 & 0.75 & 10 \\
\hline & 200 & 19.40 & 9 & 150.9 & 18.82 & 8 \\
\hline & 300 & 10.93 & 9 & 239.1 & 41.76 & 6 \\
\hline & 400 & 1.16 & 9 & 313.9 & 22.82 & 5 \\
\hline & 500 & 1.37 & 6 & 384.5 & 101.48 & 1 \\
\hline
\end{tabular}

